\title{
YIPF2 promotes chemotherapeutic agent-mediated apoptosis via enhancing TNFRSF10B recycling to plasma membrane in non-small cell lung cancer cells
}

\author{
Yingying Wang $\mathbb{D}^{1}$, Sen Guo $\mathbb{1}^{1}$, Dongmei $\mathrm{Li}^{1}$, Yongkang Tang ${ }^{1}$, Lei $\mathrm{Li}^{1}$, Ling Su${ }^{1}$ and Xiangguo Liu (1)
}

\begin{abstract}
Non-small cell lung cancer (NSCLC) is the most common histological type of lung cancer, and the identification of the apoptotic process of NSCLC is vital for its treatment. Usually, both the expression level and the cell surface level of TNFRSF10B (TNF Receptor superfamily member 10B) will increase after treatment with some chemotherapeutic agents, which plays a critical role in the apoptosis induction. However, the exact molecular mechanism underlying TNFRSF10B regulation remains largely elusive. Here, we found that TNFRSF10B, along with a vesicular trafficking regulator protein, YIPF2, were upregulated after treatment with pemetrexed (PEM) in NSCLC cells. Besides, YIPF2 increased the surface level of TNFRF10B, while YIPF2 knockdown inhibited the upregulation of TNFRSF10B and its recycling to plasma membrane. In addition, RAB8 decreased the cell surface TNFRSF10B by promoting its removing from plasma membrane to cytoplasm. Furthermore, we found that YIPF2, RAB8 and TNFRSF10B proteins interacted physically with each other. YIPF2 could further inhibit the physical interaction between TNFRSF10B and RAB8, thereby suppressing the removing of TNFRSF10B from plasma membrane to cytoplasm mediated by RAB8 and maintaining its high level on cell surface. Finally, using bioinformatics database, the YIPF2-TNFRSF10B axis was confirmed to be associated with the malignant progression of lung cancer. Taken together, we show that YIPF2 promotes chemotherapeutic agentmediated apoptosis via enhancing TNFRSF10B recycling to plasma membrane in NSCLC cells. These findings may be beneficial for the development of potential prognostic markers of NSCLC and may provide effective treatment strategy.
\end{abstract}

\section{Introduction}

Nearly $85 \%$ of primary lung cancers are of the nonsmall-cell lung cancer (NSCLC) type worldwide, and most patients present with advanced or metastatic disease at diagnosis $^{1}$. Apoptotic-associated receptor TNFRSF10B (also known as DR5 or TRAIL-R2) has been reported to play an important role in the apoptosis of various cancer cells $^{2-4}$. It locates at the cell surface and is activated upon

\footnotetext{
Correspondence: Ling Su (suling@sdu.edu.cn) or Xiangguo Liu (xgliu@sdu.edu.cn)

${ }^{1}$ Shandong Provincial Key Laboratory of Animal Cell and Developmental Biology, School of Life Sciences, Shandong University, Qingdao, China Edited by A. Stephanou
}

binding to its ligand TRAIL (tumor necrosis factor-related apoptosis inducing ligand) or aggregation induced by some agonistic antibodies such as Apomab, Tigatuzumab and TRA $-8^{5-8}$. In addition, the activation or overexpression of TNFRSF10B will signal apoptosis through CASP8-mediated activation of caspase cascades ${ }^{9}$. The elevated expression level and the cell surface level of TNFRSF10B induced by some chemotherapeutic agents play a critical role during the apoptosis of tumor cells perhaps in a ligand-independent manner. Our previous study indicated that pemetrexed (PEM), an inhibitor of folic acid synthesis used for the treatment of NSCLC patients currently ${ }^{10,11}$, can induce apoptosis by 
upregulating the expression of TNFRSF10B in NSCLC cells $^{12}$. Therefore, as an important mediator of the extrinsic apoptotic signaling pathway, TNFRSF10B has been attracted much more attention on cancer therapy ${ }^{13-15}$. Currently, most of the studies around the molecular mechanism underlying TNFRSF10B regulation were focused on the transcriptional regulation ${ }^{16,17}$. Reports indicate that proteins involved in endoplasmic reticulum stress, such as DDIT3, ATF4, TP53 and ATF3, can modulate TNFRSF10B expression at the transcriptional level ${ }^{4,18,19}$. Considering as a membrane receptor, here we propose that the vesicle transport of TNFRSF10B from the cytoplasm to plasma membrane should also be critical for its function.

YIPF2 is a member of YIP family whose name refers to the Ypt (yeast RAB GTPase)-interacting protein ${ }^{20-22}$. The YIP family proteins are predicted to have five transmembrane segments with an $\mathrm{N}$-terminal exposed to the cytoplasm and a short C-terminal exposed to the lumen of the secretory pathway ${ }^{22}$. However, the detailed function of YIPF2 is still unclear due to limited research. YIPF2 has been reported to mainly locate in the trans-Golgi network (TGN) co-localized with virous RAB proteins, suggesting that it is potentially involved in vesicle transport ${ }^{23,24}$. Besides, YIPF2 can serve as the GDF (GDI-displacement factor) of RAB5/RAB22A, thus catalyzing the dissociation of RAB-GDI complexes and regulating CD147 endocytic recycling finally in hepatocellular carcinoma cells. In addition, RAB8, one member of RAB small $G$ protein family, plays a role in membrane traffic between the TGN and the basolateral plasma membrane in many cells ${ }^{25,26}$. And the depletion of RAB8 inhibits the transport of transferrin (Tf) and $\mathrm{Tf}$ receptor (TfR) to the endocytic recycling compartment (ERC), thereby regulating the recovery of the TfR in fibrosarcoma cells ${ }^{27,28}$.

In this study, we attempt to explore the mechanism of chemotherapeutic agents-induced upregulation of TNFRSF10B and apoptosis in NSCLC cells, which will be beneficial for identifying the potential prognostic marker of NSCLC and developing effective treatment strategy.

\section{Results}

\section{PEM induces YIPF2 upregulation and apoptosis in NSCLC cells}

In clinical practice, PEM has become a preferential drug for patients with NSCLC, which can induce apoptosis of cells $^{10,12,29}$. Here, we treated the NSCLC cell lines H1792 and H1299 with PEM at different concentrations. Western blot assays showed that YIPF2, a protein which is potentially associated with vesicular transport, was markedly increased after the treatment with PEM (Fig. 1a, b). Besides, as shown in Fig. 1c, overexpression of YIPF2 in H1299 cells increased the levels of cleaved CASP8, CASP3 and PARP1, which were markers of extrinsic apoptosis pathway induced by PEM. In contrast, knockdown of YIPF2 expression in A549 cells decreased cleavage of the above proteins induced by PEM (Fig. 1d). Taken together, these data indicate that PEM upregulates levels of YIPF2 in NSCLC cells, and YIPF2 can further enhance PEM-induced apoptosis.

\section{PEM promotes apoptosis of NSCLC cells via YIPF2-TNFRSF10B axis}

It has been reported that YIPF2 and its family members are potentially involved in intracellular vesicular transport,

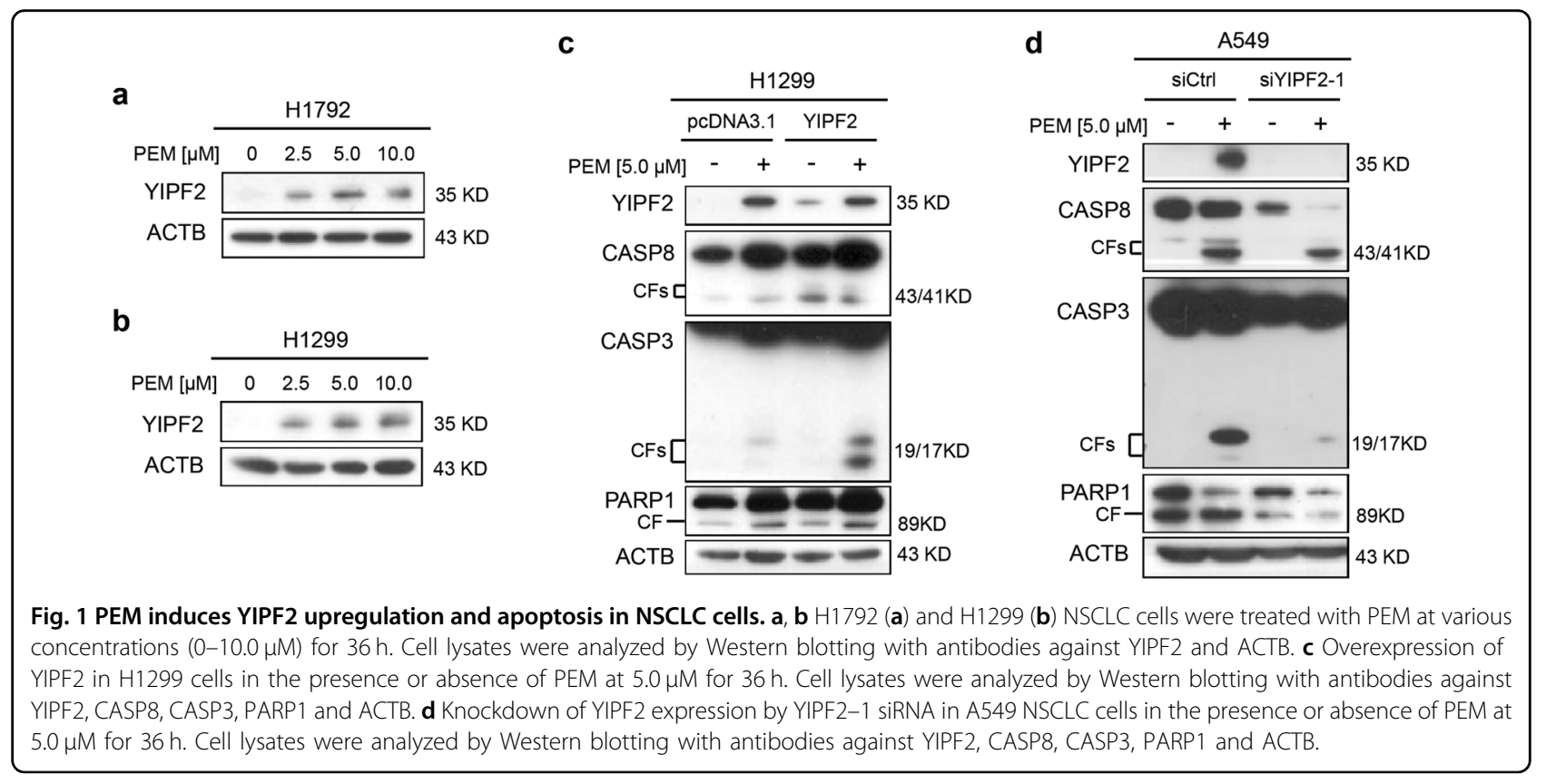


and knockdown of its expression promotes the migration of hepatocellular carcinoma cells ${ }^{23,30}$. However, it has not been identified whether YIPF2 mediates apoptosis in NSCLC cells. Here, A549 and H1792 cells were treated with 5.0 $\mu \mathrm{M}$ PEM for various times. Western blot analysis revealed that the expression of TNFRSF10B was also markedly increased compared with the controls, similar to the change of YIPF2 (Fig. 2a, b). In addition, another chemotherapeutic agent doxorubicin (DOX) was also found to upregulate the expression of YIPF2 and TNFRSF10B by western blot assays (Fig. 2c). As shown in Fig. 2d, e, western blot analysis revealed that levels of TNFRSF10B were significantly increased in YIPF2overexpressed H1792 and H1299 cells compared with the controls, whereas knockdown of YIPF2 expression in A549 and H1792 cells led to opposite effects. However, PEM treatment or changing YIPF2 levels did not affect the expression of TNFRSF10A, which was TNF receptor superfamily member 10A (Fig. 2f). In addition, rescue experiment assays indicated that PEM-induced cleavages of CASP8 and CASP3 were greatly increased after overexpression of YIPF2 in A549 cells compared with that in control cells, whereas knockdown of TNFRSF10B expression in these cells simultaneously decreased the effects of PEM-induced cleavages of CASP8 and CASP3 (Fig. 2g). On the contrary, PEM-induced cleavages of CASP8 and CASP3 were greatly decreased after knockdown of YIPF2 expression in H1299 cells compared with the control cells, whereas overexpression of TNFRSF10B in these cells simultaneously increased the effects (Fig. 2h). These data above suggest that PEM induces apoptosis of NSCLC cells via YIPF2-TNFRSF10B axis.

\section{YIPF2 enhances TNFRSF10B recycling to plasma membrane}

It is well known that TNFRSF10B is mainly localized on the plasma membrane and acts as an apoptotic receptor to induce cell apoptosis. In order to investigate how YIPF2 affects the homeostasis of TNFRSF10B, we overexpressed YIPF2 in A549 and H1792 cells. The flow cytometry analysis showed that the levels of TNFRSF10B on the plasma membrane were upregulated compared with the control treatment (Fig. 3a). Besides, the cell surface expression of TNFRSF10B was markedly decreased after knockdown of YIPF2 in A549 cells (Fig. 3b). The RT-qPCR analysis revealed that overexpression of YIPF2 in H1792 and H1299 cells did not substantially alter the mRNA levels of TNFRSF10B (Fig. 3c). Similarly, knockdown of YIPF2 expression in the above two cells still did not change the mRNA levels of TNFRSF1OB (Fig. 3d). Next, H1299 cells were treated with $10 \mu \mathrm{g} / \mathrm{ml}$ cycloheximide (CHX) for various times to inhibit new protein translation and examine the turnover of TNFRSF10B protein. Figure 3e showed increased stability of TNFRSF10B protein after YIPF2 overexpression compared with controls in H1299 cells, whereas Fig. 3f revealed stability of TNFRSF10B protein was decreased after YIPF2 knockdown compared with controls in A549 cells. These results were further confirmed by quantitative analysis (Fig. 3e, f). Altogether, these data suggest that YIPF2 enhances TNFRSF10B recycling to plasma membrane.

\section{RAB8 suppresses PEM-induced apoptosis of NSCLC cells by promoting the removing of TNFRSF10B from plasma membrane to cytoplasm}

RAB8, one member of RAB small G protein family, has been reported to mediate protein recovery from the plasma membrane to the cytosol ${ }^{27,28}$. Interestingly, the surface level of TNFRSF10B was increased in RAB8 knocked-down H1792 and A549 cells compared with cells of control as detected by flow cytometry (Fig. 4a, b). Similarly, the level of TNFRSF10B on plasma membrane was also increased after the treatment of PEM (Fig. 4a, b). Furthermore, western blot assays showed that overexpression of RAB8 in H1299 cells decreased the level of TNFRSF10B and cleaved CASP8, CASP3 and PARP1, which were markers of extrinsic apoptosis pathway induced by PEM (Fig. 4c). In contrast, knockdown of RAB8 in H1792 cells increased the level of TNFRSF10B and the cleavage of CASP8, CASP3 and PARP1 induced by PEM (Fig. 4d). In summary, these results demonstrate that RAB8 inhibits PEM-induced apoptosis of NSCLC cells by promoting the removing of TNFRSF10B from plasma membrane to cytoplasm.

\section{YIPF2 inhibits the interaction between TNFRSF10B and RAB8}

YIPF2 belongs to the YIP protein family, which has been reported to bind to $\mathrm{RAB}$ proteins to regulate vesicular transport $^{22,31}$. Thus, we performed co-immunoprecipitation (co-IP) assays to investigate why YIPF2 increased the surface expression of TNFRSF10B. Briefly, H1299 cells were transfected with pcDNA3.1 or pcDNA3.1-Flag-TNFRSF10B (short isoform) plasmids. The co-IP assays revealed that TNFRSF10B could certainly interact with endogenous YIPF2 physically (Fig. 5a). In addition, YIPF2 could also interact with endogenous TNFRSF10B in H1792 cells transfected with pEGFP-N1 or pEGFP-N1-YIPF2 plasmids (Fig. 5b). Using similar methods, we further verified the interaction between YIPF2 and endogenous RAB8 in H1792 cells (Fig. 5c). The interaction between RAB8 and endogenous TNFRSF10B was also confirmed in H1792 cells by co-IP assays (Fig. 5d). Finally, RAB8 and YIPF2 were overexpressed in $\mathrm{H} 1792$ cells, and co-IP assays revealed that YIPF2 reduced the interaction between RAB8 and endogenous TNFRSF10B in H1792 cells compared with that in control cells (Fig. 5e). Taken together, these results suggest that YIPF2, RAB8 and TNFRSF10B proteins interact with 
a

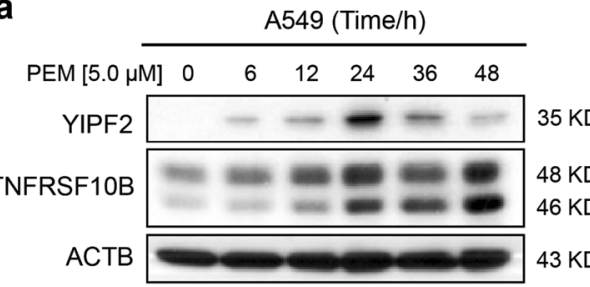

b

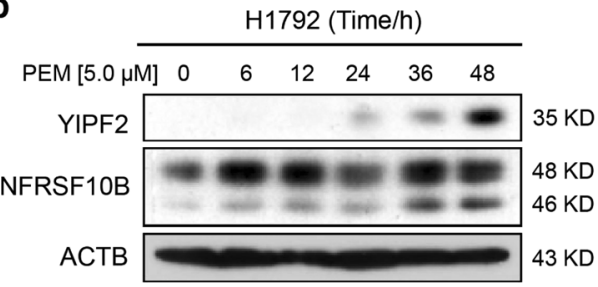

C

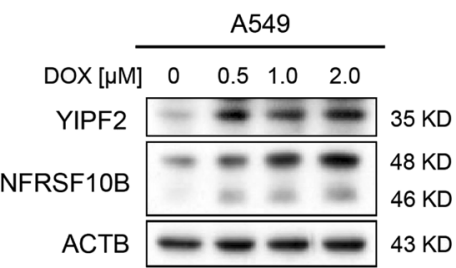

g

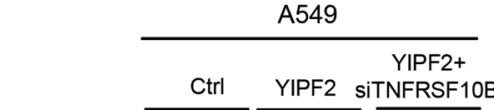

$\operatorname{PEM}[5.0 \mu \mathrm{M}]-+\frac{+}{-+}$

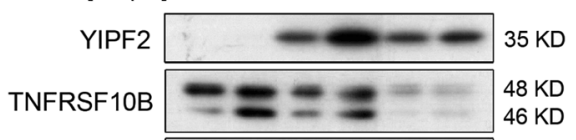

CASP8
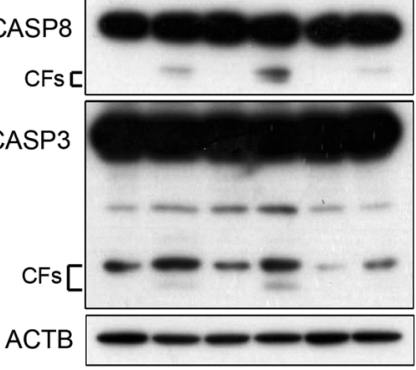

d

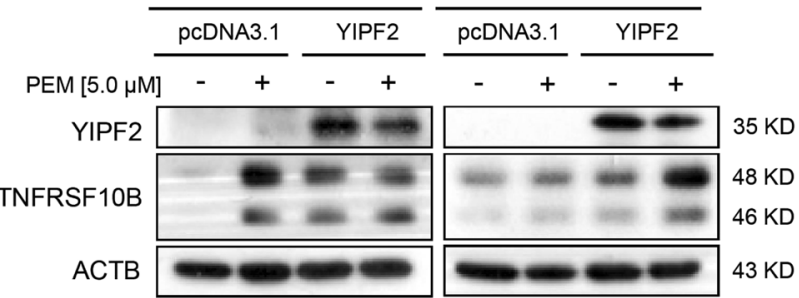

e
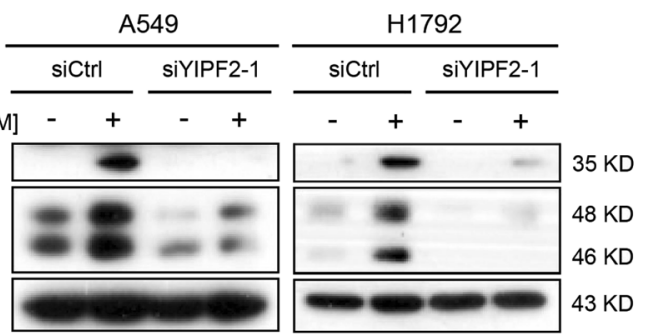

f
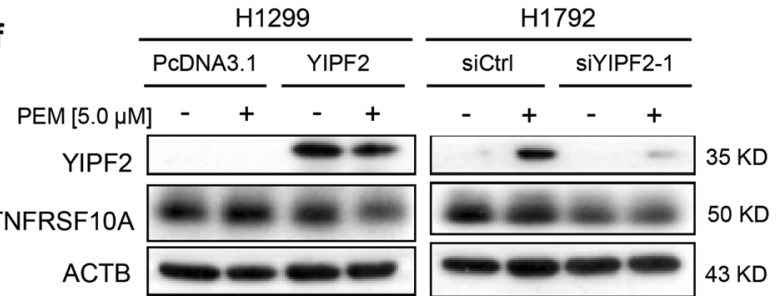

h

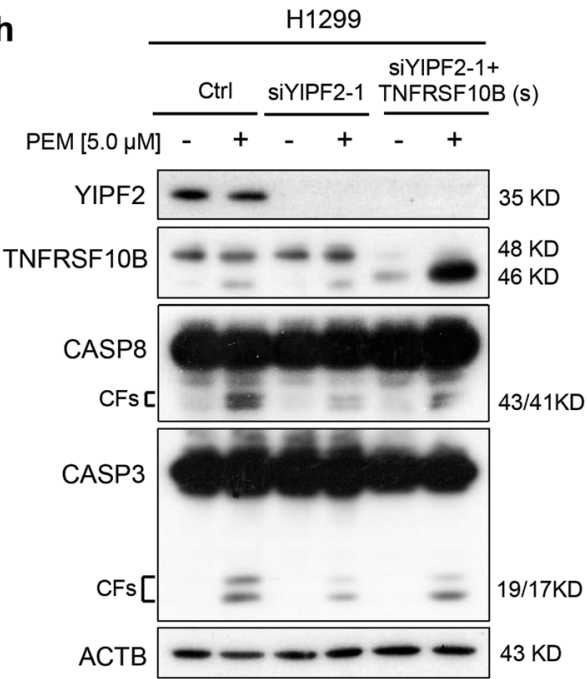

Fig. 2 PEM induces apoptosis of NSCLC cells via YIPF2-TNFRSF10B axis. a, b A549 (a) and H1792 (b) cells were treated with PEM at $5.0 \mu M$ for the indicated times $(0,6,12,24,36$ and $48 \mathrm{~h})$. Cell lysates were analyzed by Western blotting with antibodies against YIPF2, TNFRSF10B and ACTB. c A549 cells were treated with doxorubicin (DOX) at various concentrations (0-2.0 $\mu \mathrm{M})$ for $18 \mathrm{~h}$. Cell lysates were analyzed by Western blotting with antibodies against YIPF2, TNFRSF10B and ACTB. d Overexpression of YIPF2 in H1792 and H1299 cells in the presence or absence of PEM at $5.0 \mu M$ for $36 \mathrm{~h}$. Cell lysates were analyzed by Western blotting with antibodies against YIPF2, TNFRSF10B and ACTB. e Knockdown of YIPF2 expression by YIPF2-1 siRNA in A549 and H1792 cells in the presence or absence of PEM at $5.0 \mu \mathrm{M}$ for $36 \mathrm{~h}$. Cell lysates were analyzed by Western blotting with antibodies against YIPF2, TNFRSF10B and ACTB. f Overexpression of YIPF2 in H1299 cells (left) or Knockdown of YIPF2 expression by YIPF2-1 siRNA in $\mathrm{H} 1792$ cells (right) in the presence or absence of PEM at $5.0 \mu \mathrm{M}$ for $36 \mathrm{~h}$. Cell lysates were analyzed by Western blotting with antibodies against YIPF2, TNFRSF10A and ACTB. $\mathbf{g}$ Three A549 cell lines (Ctrl, YIPF2, YIPF2 + siTNFRSF10B) in the presence or absence of PEM at $5.0 \mu \mathrm{M}$ for $36 \mathrm{~h}$. Cell lysates were analyzed by Western blotting with antibodies against YIPF2, TNFRSF10B, CASP8, CASP3 and ACTB. $\mathbf{h}$ Three H1299 cell lines (Ctrl, siYIPF2-1, siYIPF2-1 + TNFRSF10B (short isoform)) in the presence or absence of PEM at $5.0 \mu \mathrm{M}$ for $36 \mathrm{~h}$. Cell lysates were analyzed by Western blotting with antibodies against YIPF2, TNFRSF10B, CASP8, CASP3 and ACTB. 


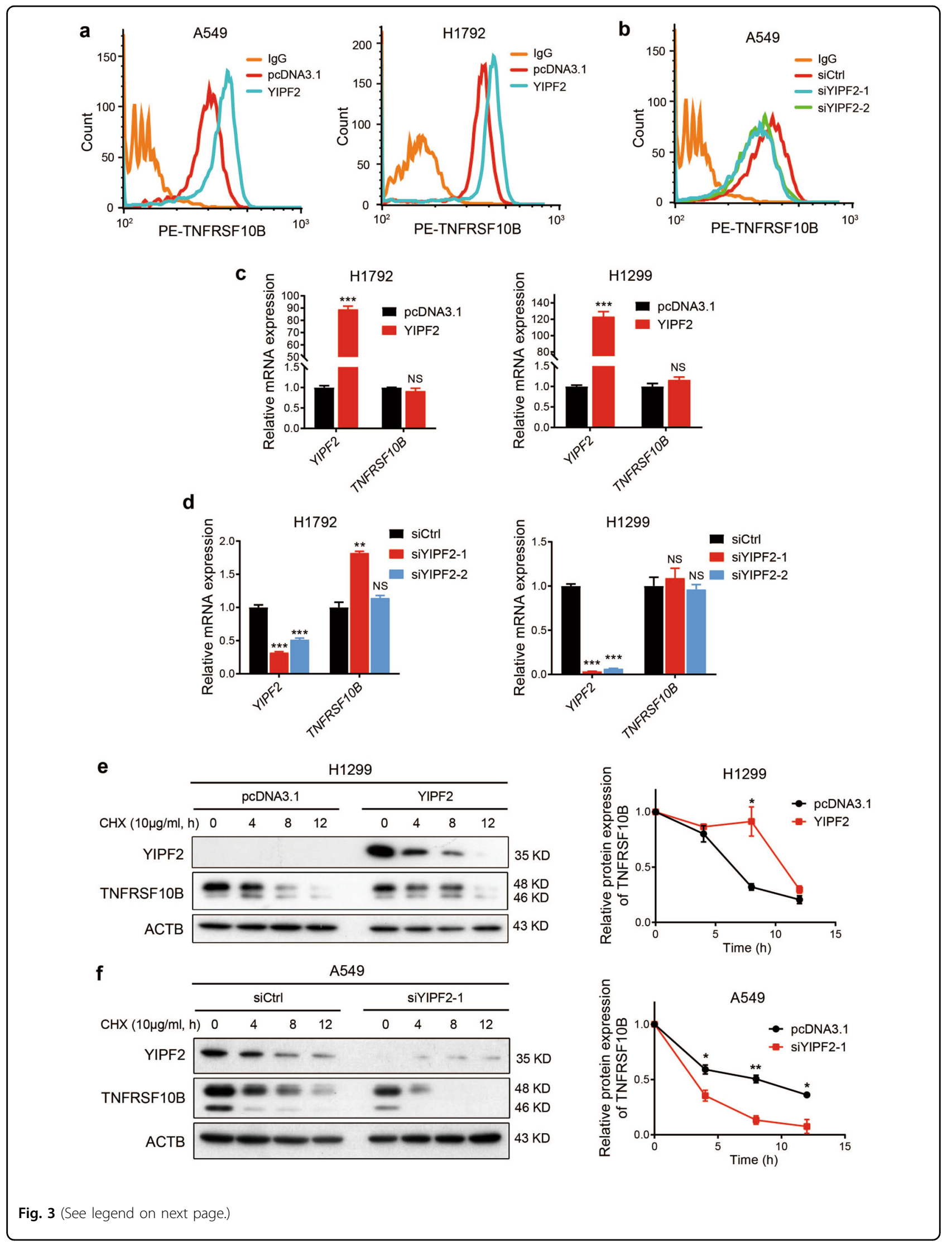


(see figure on previous page)

Fig. 3 YIPF2 enhances TNFRSF10B recycling to plasma membrane. a Overexpression of YIPF2 in A549 and H1792 cells. The surface expression of TNFRSF10B was confirmed by flow cytometry analyses. $\mathbf{b}$ Knockdown of YIPF2 expression by YIPF2-1 and YIPF2-2 siRNA in A549 cells. The surface expression of TNFRSF10B was confirmed by flow cytometry analyses. c Relative RT-qPCR analyses of YIPF2 and TNFRSF10B mRNA levels after YIPF2 overexpression in $\mathrm{H} 1792$ (left) and H1299 (right) cells $(n=3)$. d Relative RT-qPCR analyses of YIPF2 and TNFRSF1OB mRNA levels after YIPF2 knocking down in H1792 (left) and H1299 (right) cells $(n=3)$. e Left: Overexpression of YIPF2 in H1299 cells in the presence or absence of cycloheximide (CHX) at $10 \mu \mathrm{g} / \mathrm{ml}$ for the indicated times (0, 4, 8 and $12 \mathrm{~h}$ ). Cell lysates were analyzed by Western blotting with antibodies against YIPF2, TNFRSF10B and ACTB. Right: The band intensity of TNFRSF10B was quantified by ImageJ software and plotted. This experiment was repeated three times independently with similar results. $\mathbf{f}$ Left: Knockdown of YIPF2 expression by YIPF2-1 siRNA in A549 cells in the presence or absence of cycloheximide (CHX) at $10 \mu \mathrm{g} / \mathrm{ml}$ for the indicated times $(0,4,8$ and $12 \mathrm{~h})$. Cell lysates were analyzed by Western blotting with antibodies against YIPF2, TNFRSF10B and ACTB. Right: The band intensity of TNFRSF10B was quantified by ImageJ software and plotted. This experiment was repeated three times independently with similar results. (mean $\pm \mathrm{SEM}, n=3$ independent experiments; NS, not significant; ${ }^{*} P<0.05,{ }^{* *} P<0.01$ and ${ }^{* *} P<0.001 ; P$-values in $\mathbf{c}$, e and $\mathbf{f}$ were obtained using two-tailed Student's $t$-tests, $P$-values in $\mathbf{d}$ were obtained using one-way ANOVA followed by Bonferroni's post-hoc test).

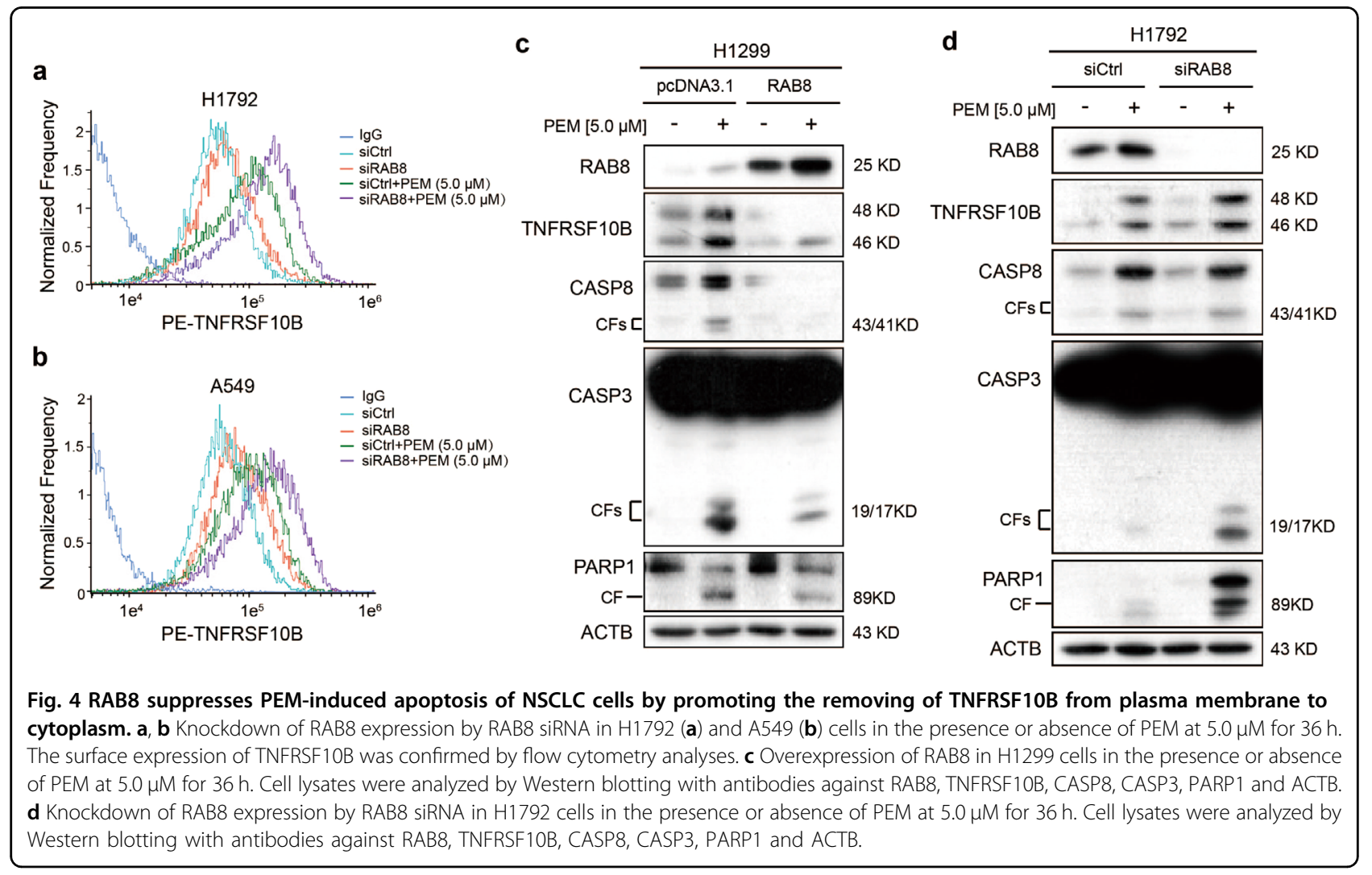

each other, and YIPF2 inhibits the interaction between TNFRSF10B and RAB8, thereby suppressing the removing of TNFRSF10B from plasma membrane to cytoplasm through RAB8 and maintaining its high level on plasma membrane.

\section{YIPF2 and TNFRSF10B are associated with malignant progression in lung cancer patients}

To determine whether our findings are clinically relevant, two Oncomine datasets, namely TCGA Lung 2 and Weiss Lung, were used to examine the mRNA expression of YIPF2 (Fig. 6a). The data showed that the mRNA levels of YIPF2 were significantly lower in lung adenocarcinoma tissues than that in normal tissues. Similarly, mRNA expression of TNFRSF1OB was also lower in lung adenocarcinoma tissues than that in normal tissues in two Oncomine datasets (TCGA Lung 2 and Bhattacharjee Lung) (Fig. 6b). Using the Kaplan-Meier method followed by the log-rank test, we further confirmed that higher expression of YIPF2 was correlated with higher firstprogression survival (FPS, upper) and post-progression survival (PPS, lower) in chemotherapy-treated patients (Fig. 6c). Similarly, higher TNFRSF10B mRNA levels were also correlated with higher first-progression survival (FPS, 


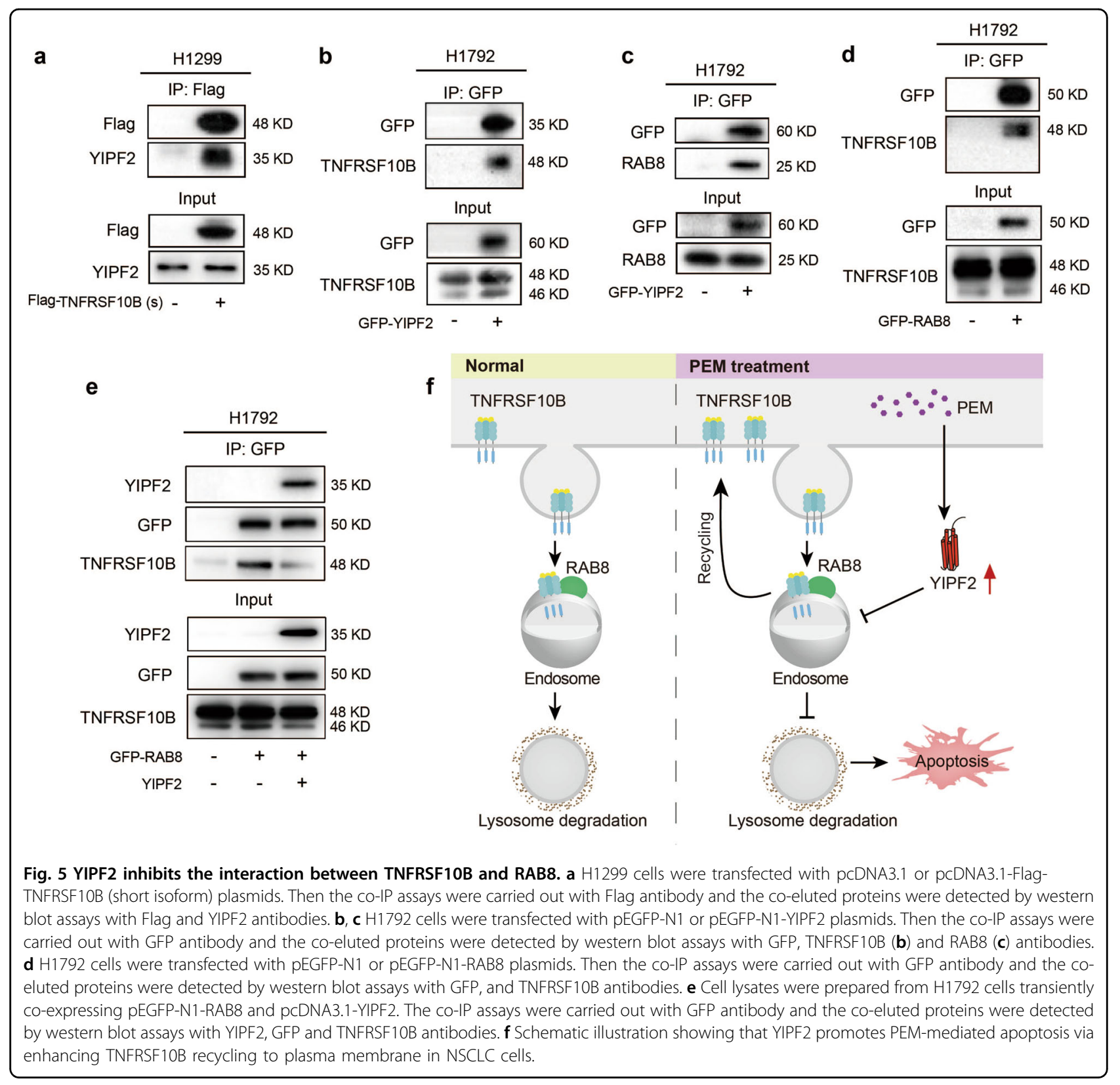

upper) and post-progression survival (PPS, lower) in chemotherapy-treated patients (Fig. 6d). Finally, YIPF2 expression tended to be positively associated with the expression of TNFRSF10B in two GEO datasets (GDS1688 and GDS3627), which contained 29 lung cancer cell lines and 58 NSCLC cell lines respectively (Fig. 6e). Collectively, these data reveal that the mRNA expression of YIPF2 and TNFRSF1OB is associated with malignant progression in lung cancer patients.

\section{Discussion}

Currently, there are many studies focusing on the proliferation and apoptosis of NSCLC cells, aiming to obtain more effective treatments ${ }^{32}$. Randomized trials show that PEM has a good therapeutic effect and has become a preferential drug for patients with NSCLC ${ }^{33,34}$. Three enzymes used in purine and pyrimidine synthesis will be blocked by PEM, which are thymidylate synthase (TS), dihydrofolate reductase (DHFR) and glycinamide ribonucleotide formyltransferase $\left(\right.$ GARFT) ${ }^{35}$. Thus, PEM treatment inhibits the cellular DNA machinery via disruption of folic acid metabolism, thereby preventing cellular division and replication and causing cell cycle arrest and apoptosis ${ }^{12,29,36}$.

In the study, we found that the expression of YIPF2 was increased after PEM treatment, and its overexpression could further promote PEM-induced apoptosis in NSCLC 


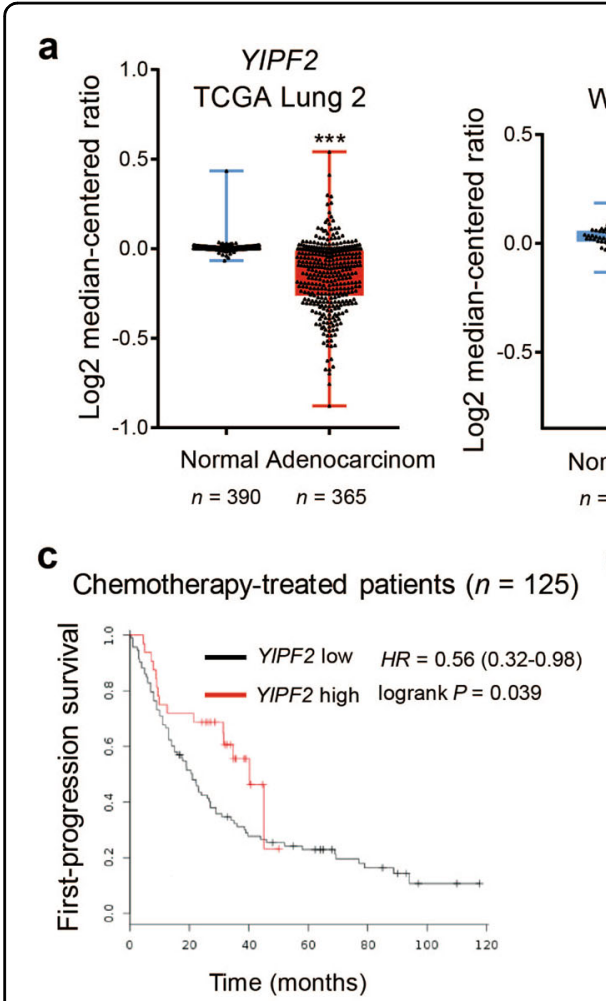

Chemotherapy-treated patients $(n=88)$

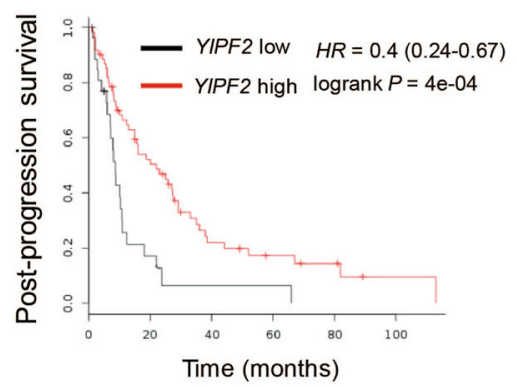

YIPF2

Weiss Lung
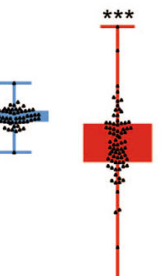

ormal Adenocarcinom $n=59 \quad n=77$ b
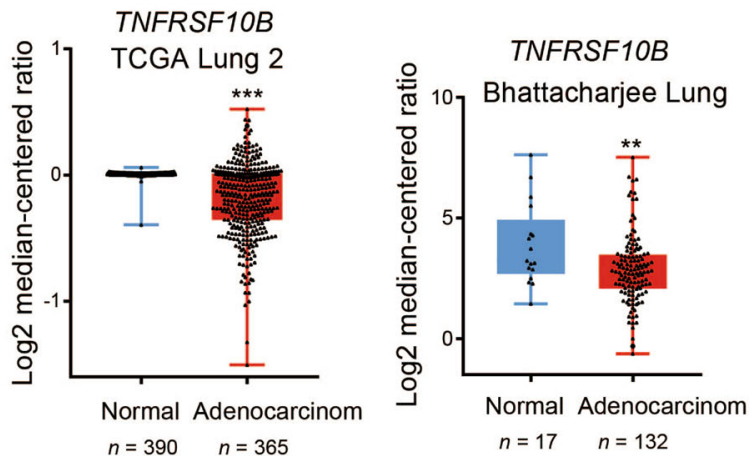

d
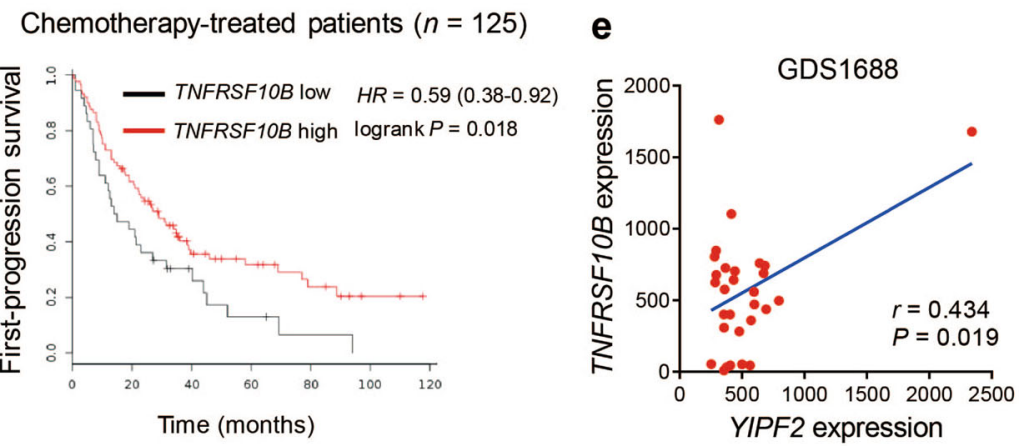

Chemotherapy-treated patients $(n=88)$
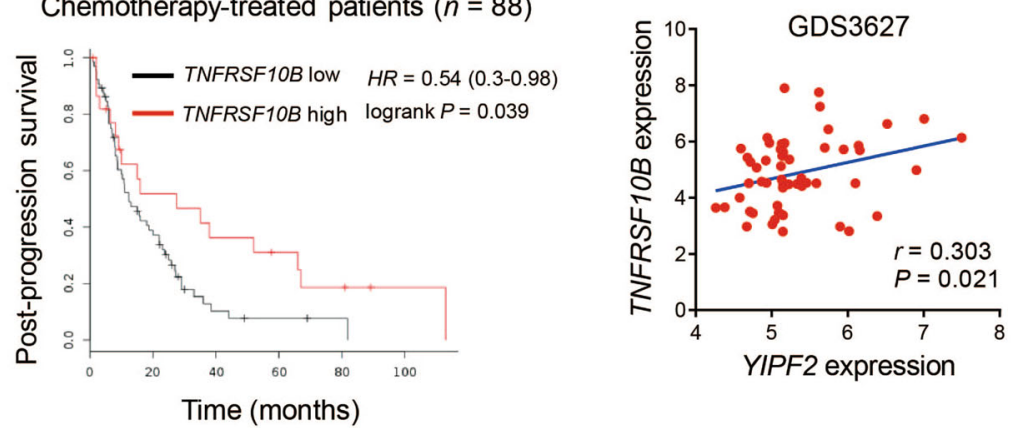

Fig. 6 YIPF2 and TNFRSF10B are associated with malignant progression in lung cancer patients. a Box plots of YIPF2 mRNA levels determined from two Oncomine datasets, namely TCGA Lung 2 and Weiss Lung ${ }^{* *} P<0.01$ and ${ }^{* *} P<0.001 ; P$-values were obtained using two-tailed Student's $t$ tests). $\mathbf{b}$ Box plots of TNFRSFIOB mRNA levels determined from two Oncomine datasets, namely TCGA Lung 2 and Bhattacharjee Lung. c Kaplan-Meier plots of the first-progression survival (FPS, upper) and post-progression survival (PPS, lower) of chemotherapy-treated patients stratified by YIPF2 expression. The data were acquired from the Kaplan-Meier plotter database ( $P$-values were obtained using the log-rank test). $\mathbf{d}$ Kaplan-Meier plots of the first-progression survival (FPS, upper) and post-progression survival (PPS, lower) of chemotherapy-treated patients stratified by TNFRSF1OB expression. The data were acquired from the Kaplan-Meier plotter database ( $P$-values were obtained using the log-rank test). e Scatter plots showing the correlation of YIPF2 expression with TNFRSF1OB expression in lung cancer cells in two GEO datasets (upper: GDS1688 which contains 29 lung cancer cell lines; lower: GDS3627 which contains 58 NSCLC cell lines). The $r$ value was calculated via Spearman's rank correlation coefficient analysis.

cells. YIPF2 belongs to YIP family which has been reported to interact with RAB small G protein and plays vital roles in intracellular vesicular transport ${ }^{20,22,24,31,37}$. Numerous reports have discovered that some chemotherapeutic agents including PEM can induce endoplasmic reticulum (ER) stress in tumor cells ${ }^{12,38}$. We found that the expression of YIPF2, along with a hallmark transcription factor of ER stress, XBP1S, were upregulated after treatment with ER stress-inducing agents, thapsigargin (TG) and tunicamycin (TM) in NSCLC cells (data not shown). Furthermore, YIPF2 was conformed to be the target gene of XBP1S which binds to the promoter region of YIPF2 (data not shown), suggesting that chemotherapeutic agents mediated upregulation of YIPF2 potentially via ER stress pathway. This project requires further experimental verification in the future. 
A key finding of our study is that the elevated YIPF2 maintains high levels of TNFRSF10B on cell surface via inhibiting its removing from plasma membrane to cytoplasm mediated by RAB8. It is well known that two major apoptotic signaling pathways exist in cells: the intrinsic mitochondria-mediated pathway and the extrinsic death receptor-induced pathway, and TNFRSF10B is an important mediator of the extrinsic apoptotic signaling pathway ${ }^{39}$. As a membrane receptor, vesicle transport of TNFRSF10B between cytoplasm and plasma membrane is critical for its function and should be paid more attention. However, there are rare researches about the vesicle transport of TNFRSF10B in the literature. It has been reported that the translocation of TNFRSF10B from TGN to plasma membrane is regulated by cargo transport proteins, such as ARF1, RHO GAP protein ARAP1 ${ }^{40}$ and nuclear translocation signaling proteins ${ }^{41}$. In addition, the faulty trafficking of TNFRS10B to plasma membrane will lead to its deficiency on cell surface and accumulation in cytosol $^{41}$, $\mathrm{TGN}^{40}$ and nuclear perimeter ${ }^{42}$. On the contrary, the internalization of TNFRSF10B will occur upon binding to its ligand TRAIL, initiating extrinsic death receptorinduced apoptotic pathway. It has been reported that the endocytosed TNFRSF10B will be trafficked to the lysosome by RAB7 for degradation ${ }^{43}$. As a result, lysosomal degradation prevents receptor recycling back to the plasma membrane for further TRAIL ligation.

We also found that the surface level of TNFRSF10B was increased in RAB8 knockdown NSCLC cells, and RAB8 interacted with TNFRSF10B and YIPF2, suggesting that RAB8 participates in membrane trafficking of TNFRSF10B potentially through promoting its removing from plasma membrane to cytoplasm. Furthermore, co-IP assays confirmed that YIPF2-induced upregulation of TNFRSF10B on cell surface is due to inhibition of the interaction between RAB8 and TNFRSF10B. Besides, using Kaplan-Meier plotter database, $R A B 8$ mRNA levels were found to be correlated with survival rates in lung cancer patients (data not shown). As a GTPase, the activity of RAB8 is regulated by guanine nucleotide exchange factors (GEFs) that mediate GTP loading, also by GTPase activating proteins (GAPs) that convert them into inactive GDP-forms. RAB8-specific GEFs contains Rabin8 and GRAB ${ }^{44-46}$. Endogenous RAB8 is found in dynamic cell structures like filopodia, lamellipodia, protrusions, ruffles, and primary cilia ${ }^{26}$. In addition, RAB8 is involved in many important processes of cells such as cell migration $^{47}$, neuron differentiation ${ }^{27}$, ciliogenesis ${ }^{48}$, epithelial polarization ${ }^{49}$, especially in membrane trafficking $^{27,50}$. The function of RAB8 to participate in membrane trafficking is exactly what our research focuses on. Endogenous RAB8 is found to associate with vesicles, macropinosomes, and tubular structures via recognition by RAB8-specific antibody ${ }^{27,44}$. Furthermore, previous studies showed that RAB8 colocalizes with ARF6, EHD1, EHD3, ITGB1, MHCI, MYO5B, and MYO5C ${ }^{27,51-53}$, indicating that it participates in a recycling pathway based on clathrin-independent endocytosis. Consistently with TNFRSF10B, it has been described that Rab8 promotes the relocalization of Huntingtin protein to vesicles ${ }^{54}$.

In summary, we found that the expression of YIPF2 is increased after PEM treatment, which could promote the recycling of TNFRSF10B to plasma membrane by inhibiting the interaction between TNFRSF10B and RAB8, thus enhancing apoptosis of NSCLC cells eventually (Fig. 5f). Our findings provide a novel insight into the mechanism underlying TNFRSF10B regulation and will be beneficial for identifying the potential prognostic marker for NSCLC treatment.

\section{Materials and methods \\ Cell lines and cell culture}

The human NSCLC cell lines H1792, H1299 and A549 were originally obtained from the American Type Culture Collection (ATCC). They were cultured in RPMI 1640 medium with $10 \% \mathrm{FBS}$ at $37{ }^{\circ} \mathrm{C}$ in a humidified atmosphere consisting of $5 \% \mathrm{CO} 2$ and tested for no mycoplasma contamination.

\section{Reagents and antibodies}

PEM was purchased from Sigma-Aldrich. CHX was purchased from MedChem Express. The TNFRSF10B antibody used in flow cytometry was obtained from Thermo Fisher Scientific (Cat. no. 12-9908-42; eBioscience). The primary antibodies used in western blot assays and Immunoprecipitation were as follows: anti-YIPF2 (Cat. no. HPA019902; Sigma-Aldrich), ACTB (Cat. no. A1978; Sigma-Aldrich), CASP8 (Cat. no. 9746S; CST), CASP3 (Cat. no. NB100-56708; Novus Biologicals), PARP1 (Cat. no. 9542S; CST), TNFRSF10B (Cat. no. 2019; ProSci Incorporated), RAB8 (Cat. no. 6975S; CST), Flag (Cat. no. F7425, F1804; Sigma-Aldrich) and GFP (Cat. no. G1544, SigmaAldrich; Cat. no. sc-9996, Santa Cruz).

\section{siRNA and plasmid transfection}

The cells were transfected with jetPRIME transfection reagent (Polyplus transfection) or LipoMax reagent (Sudgen Biotechnology) in serum-free Opti-MEM (Gibco) according to the instruction manual. All siRNAs were synthesized from GenePharma (Shanghai, China). The sense and anti-sense strands of siRNAs were as follows: YIPF2 siRNA-1 sense: 5'-GGCUGUAAGUUGUACUU CUTT-3' YIPF2 siRNA-1 antisense: $5^{\prime}$-AGAAGUACAA CUUACAGCCTT- $3^{\prime}$

YIPF2 siRNA-2 sense: $5^{\prime}$-GUGCCACGUUGGCCUUU GUTT-3' YIPF2 siRNA-2 antisense: 5'-ACAAAGGCCAA CGUGGCACTT- $3^{\prime}$ 
TNFRSF10B siRNA sense: $5^{\prime}$-GACCCUUGUGCUCGU UGUCTT-3' TNFRSF10B siRNA antisense: 5'-GACAAC GAGCACAAGGGUCTT- $3^{\prime}$

RAB8 siRNA sense: $5^{\prime}$ - GAGAATTAAACTGCAGATA TT-3' RAB8 siRNA antisense: 5'- GCTCGATGGCAAGA GAATTTT- $3^{\prime}$

YIPF2, TNFRSF10B and RAB8 coding regions were amplified from A549 cDNA and subcloned into pcDNA3.1 or pEGFP-N1 vector. The primers were as follows:

YIPF2 sense: 5'-CCCGCTCGAGGCCGCCACCATGG CATCGGCCGACGAGCTGACCTTCC-3' YIPF2 antisense: 5'-CCGCGGATCCCGGGAGGGGGCCAGGGAC TGCGGCAAG-3'

TNFRSF10B sense: 5'-CGGATCCGCCGCCACCATG GAACAACGGGGACAGAACGC-3' TNFRSF10B antisense: 5'-CCTCGAGTTAATGGTGATGGTGATGATG GGACATGGCAGAGTCTGCAT-3'

RAB8 sense: 5'-CGGTACCGCCGCCACCATGGCGA AGACCTACGATTAC-3' RAB8 antisense: 5'-CGGATC CCCTCACAGAAGAACACATCGG-3'

\section{Western blot analysis}

Cells were harvested and rinsed with pre-chilled PBS on ice. They were lysed in lysis buffer on ice for $30 \mathrm{~min}$ and then purified via centrifugation for $13 \mathrm{~min}$ at $4{ }^{\circ} \mathrm{C}$. Protein extracts were resolved through $8 \%-15 \%$ SDS-PAGE, transferred to PVDF membranes, and probed with primary antibodies. Peroxidase-conjugated anti-mouse or rabbit antibody (Bio-Rad Laboratories) was used as secondary antibody and the antigen-antibody reaction was visualized by enhanced chemiluminescence assay.

\section{Immunoprecipitation}

Cells were lysed in lysis buffer $(20 \mathrm{mM}$ Tris- $\mathrm{HCl}, \mathrm{pH} 7.5$; $150 \mathrm{mM} \mathrm{NaCl} ; 1 \mathrm{mM}$ Na2EDTA; $1 \mathrm{mM}$ EGTA; $2.5 \mathrm{mM}$ sodium pyrophosphate; $1 \mathrm{mM} \beta$-glycerophosphate; $1 \mathrm{mM}$ $\mathrm{Na}_{3} \mathrm{VO}_{4} ; 0.5 \%$ Triton) on ice for $30 \mathrm{~min}$ then purified via centrifugation for $15 \mathrm{~min}$ at $4{ }^{\circ} \mathrm{C}$. The supernatants were incubated with antibody at $4{ }^{\circ} \mathrm{C}$ for $1 \mathrm{~h}$. Then the mixture was incubated with protein A beads (ThermoFisher) at $4{ }^{\circ} \mathrm{C}$ for $2 \mathrm{~h}$. The beads were washed twice with $1 \mathrm{ml}$ of lysis buffer. $20 \mu \mathrm{l} 2 \times$ SDS buffer were added for elution $\left(100^{\circ} \mathrm{C}\right.$, $10 \mathrm{~min}$ ). Samples were centrifuged for western blot analysis.

\section{Flow cytometry}

To assay the expression of TNFRSF10B on the plasma membrane, $10^{6}$ cells were incubated at room temperature for $30 \mathrm{~min}$ with $5 \mu \mathrm{l}$ of nonspecifc isotype-matched control IgG and $5 \mu \mathrm{l}$ of mouse monoclonal antibodies conjugated with PE fluorochrome (Cat. no. 12-9908-42; eBioscience). Unbound antibodies were removed by washing the cells twice in PBS buffer. Analysis was performed on a Guava EasyCyte flow cytometer (Merck Millipore), and data were processed and presented using FlowJo software.

\section{RT-qPCR}

Reverse transcription-quantitative real-time PCR (RTqPCR) was performed with a LightCycler 480 System (Roche Diagnostics), using Real-Time PCR Super Mix (Mei5Bio, China) according to the manufacturer's instruction. All reactions were done in a $20 \mu \mathrm{l}$ reaction volume in triplicate. Primers were obtained from Sangon Biotech. Following an initial denaturation at $95^{\circ} \mathrm{C}$ for $30 \mathrm{~s}, 40$ cycles of PCR amplification were performed at $95^{\circ} \mathrm{C}$ for $5 \mathrm{~s}$ and $60^{\circ} \mathrm{C}$ for $30 \mathrm{~s}$. Standard curves were generated and the relative amount of target gene mRNA was normalized to GAPDH. The primer sequences are as following:

YIPF2 forward: $5^{\prime}$-TTCGAGGAGGCCACTAATCTT- $3^{\prime}$ YIPF2 reverse: $5^{\prime}$-AGTAGCTGAAGGTCCAGAATCC-3' TNFRSF10B forward: 5'-GCCCCACAACAAAAGAGG TC-3' TNFRSF1OB reverse: 5'-AGGTCATTCCAGTGA GTGCTA-3'

GAPDH forward: 5'-ACGGATTTGGTCGTATTGGG-3' GAPDH reverse: 5'-CGCTCCTGGAAGATGGTGAT-3'

\section{Statistics}

All statistical analyses were performed using SPSS for Windows version 13.0 (SPSS). Two-tailed Student's $t$ tests were used for comparisons between two groups, and one-way ANOVA followed by Bonferroni's posthoc test was used for multiple comparisons (three or more groups). The Kaplan-Meier curves for survival analyses were determined using the log-rank test. Spearman's rank correlation coefficient analysis was performed to assess the correlation of YIPF2 expression with TNFRSF10B expression in GEO datasets. All experiments for cell cultures were performed independently at least three times and in triplicate each time. In all cases, $P$-values $<0.05$ were considered statistically significant.

\begin{abstract}
Acknowledgements
This work was supported by grants from National Natural Science Foundation of China (31571422, 31771526, 81672855, 81902994), Shandong Provincial Key Laboratory of Animal Cell and Developmental Biology (SPKLACDB-2019012, SPKLACDB-2019017), Shandong Special Construction Program for Provincial Key Laboratory (SDKL2018017) and The Fundamental Research Funds of Shandong University (2019GN060). We thank Zhifeng Li from SKLMT (State Key Laboratory of Microbial Technology, Shandong University) for the assistance in flow cytometry analyses
\end{abstract}

Conflict of interest

The authors declare that they have no conflict of interest.

\section{Publisher's note}

Springer Nature remains neutral with regard to jurisdictional claims in published maps and institutional affiliations.

Received: 18 December 2019 Revised: 19 March 2020 Accepted: 20 March 2020

Published online: 17 April 2020 


\section{References}

1. Midha, A., Dearden, S. \& McCormack, R. EGFR mutation incidence in nonsmall-cell lung cancer of adenocarcinoma histology: a systematic review and global map by ethnicity (mutMapll). Am. J. Cancer Res. 5, 2892-2911 (2015).

2. Abdelrahim, M., Newman, K., Vanderlaag, K., Samudio, I. \& Safe, S. 3,3'-diindolylmethane (DIM) and its derivatives induce apoptosis in pancreatic cancer cells through endoplasmic reticulum stress-dependent upregulation of DR5. Carcinogenesis 27, 717-728 (2006).

3. He, K., Zheng, X., Li, M., Zhang, L. \& Yu, J. mTOR inhibitors induce apoptosis in colon cancer cells via CHOP-dependent DR5 induction on 4E-BP1 dephosphorylation. Oncogene 35, 148-157 (2016).

4. Chen, P. et al. Neddylation inhibition activates the extrinsic apoptosis pathway through ATF4-CHOP-DR5 axis in human esophageal cancer cells. Clin. Cancer Res. 22, 4145-4157 (2016).

5. Adams, C. et al. Structural and functional analysis of the interaction between the agonistic monoclonal antibody Apomab and the proapoptotic receptor DR5. Cell Death Differ. 15, 751-761 (2008).

6. Forero-Torres, A. et al. Phase I trial of weekly tigatuzumab, an agonistic humanized monoclonal antibody targeting death receptor 5 (DR5). Cancer Biother. Radiopharm. 25, 13-19 (2010).

7. Jin, H. et al. Cooperation of the agonistic DR5 antibody apomab with chemotherapy to inhibit orthotopic lung tumor growth and improve survival. Clin. Cancer Res. 14, 7733-7740 (2008).

8. Ichikawa, K. et al. Tumoricidal activity of a novel anti-human DR5 monoclonal antibody without hepatocyte cytotoxicity. Nat. Med. 7, 954-960 (2001).

9. Kelley, S. K. \& Ashkenazi, A. Targeting death receptors in cancer with Apo2L TRAIL. Curr. Opin. Pharmacol. 4, 333-339 (2004).

10. Sequist, L. V. et al. Phase III study of afatinib or cisplatin plus pemetrexed in patients with metastatic lung adenocarcinoma with EGFR mutations. J. Clin. Oncol. 31, 3327-3334 (2013).

11. Huang, X. E. et al. Pemetrexed as a component of first-, second- and third- line chemotherapy in treating patients with metastatic lung adenocarcinoma. Asian Pac. J. Cancer Prev. 14, 6663-6667 (2014).

12. $\mathrm{Su}$, L. et al. Death receptor 5 and cellular FLICE-inhibitory protein regulate pemetrexed-induced apoptosis in human lung cancer cells. Eur. J. Cancer 47, 2471-2478 (2011).

13. Yang, A., Wilson, N. S. \& Ashkenazi, A. Proapoptotic DR4 and DR5 signaling in cancer cells: toward clinical translation. Curr. Opin. Cell Biol. 22, 837-844 (2010).

14. Liu, X. et al. The proteasome inhibitor PS-341 (bortezomib) up-regulates DR5 expression leading to induction of apoptosis and enhancement of TRAILinduced apoptosis despite up-regulation of c-FLIP and survivin expression in human NSCLC cells. Cancer Res. 67, 4981-4988 (2007).

15. Liu, X., Yue, P., Zhou, Z., Khuri, F. R. \& Sun, S. Y. Death receptor regulation and celecoxib-induced apoptosis in human lung cancer cells. J. Natl. Cancer Inst. 96, 1769-1780 (2004).

16. Kim, Y. H., Park, J. W., Lee, J. Y. \& Kwon, T. K. Sodium butyrate sensitizes TRAlLmediated apoptosis by induction of transcription from the DR5 gene promoter through Sp1 sites in colon cancer cells. Carcinogenesis 25, 1813-1820 (2004).

17. Takimoto, R. \& El-Deiry, W. S. Wild-type p53 transactivates the KILLER/DR5 gene through an intronic sequence-specific DNA-binding site. Oncogene 19, 1735-1743 (2000).

18. Li, T. et al. DDIT3 and KAT2A proteins regulate TNFRSF10A and TNFRSF10B expression in endoplasmic reticulum stress-mediated apoptosis in human lung cancer cells. J. Biol. Chem. 290, 11108-11118 (2015).

19. Taketani, K. et al. Key role of ATF3 in p53-dependent DR5 induction upon DNA damage of human colon cancer cells. Oncogene 31, 2210-2221 (2012).

20. Calero, M., Winand, N. J. \& Collins, R. N. Identification of the novel proteins Yip4p and Yip5p as Rab GTPase interacting factors. FEBS Lett. 515, 89-98 (2002).

21. Kano, F. et al. Yip1A regulates the COPI-independent retrograde transport from the Golgi complex to the ER. J. Cell Sci. 122(Pt 13), 2218-2227 (2009).

22. Shakoori, A. et al. Identification of a five-pass transmembrane protein family localizing in the Golgi apparatus and the ER. Biochem. Biophys. Res. Commun. 312, 850-857 (2003).

23. Soonthornsit, J. et al. YIPF1, YIPF2, and YIPF6 are medial-/trans-Golgi and transGolgi network-localized Yip domain family proteins, which play a role in the Golgi reassembly and glycan synthesis. Exp. Cell Res. 353, 100-108 (2017).

24. Tang, B. L. et al. A membrane protein enriched in endoplasmic reticulum exit sites interacts with COPII. J. Biol. Chem. 276, 40008-40017 (2001).
25. Huber, L. A. et al. Rab8, a small GTPase involved in vesicular traffic between the TGN and the basolateral plasma membrane. J. Cell Biol. 123, 35-45 (1993).

26. Peranen, J. Rab8 GTPase as a regulator of cell shape. Cytoskeleton $\mathbf{6 8}, 527-539$ (2011).

27. Hattula, K. et al. Characterization of the Rab8-specific membrane traffic route linked to protrusion formation. J. Cell Sci. 119(Pt 23), 4866-4877 (2006).

28. Ullrich, O., Reinsch, S., Urbe, S., Zerial, M. \& Parton, R. G. Rab11 regulates recycling through the pericentriolar recycling endosome. J. Cell Biol. 135 913-924 (1996).

29. Yan, J. et al. Usp9x- and Noxa-mediated Mcl-1 downregulation contributes to pemetrexed-induced apoptosis in human non-small-cell lung cancer cells. Cell Death Dis. 5, e1316 (2014).

30. Qi, S. et al. YIPF2 is a novel Rab-GDF that enhances HCC malignant phenotypes by facilitating CD147 endocytic recycle. Cell Death Dis. 10, 462 (2019).

31. Kranjc, T. et al. Functional characterisation of the YIPF protein family in mammalian cells. Histochem. Cell Biol. 147, 439-451 (2017).

32. Li, W. et al. Cold atmospheric plasma and iron oxide-based magnetic nanoparticles for synergetic lung cancer therapy. Free Radic. Biol. Med. 130, 71-81 (2019).

33. Hanna, N. et al. Randomized phase III trial of pemetrexed versus docetaxel in patients with non-small-cell lung cancer previously treated with chemotherapy. J. Clin. Oncol. 22, 1589-1597 (2004).

34. Scagliotti, G. et al. The differential efficacy of pemetrexed according to NSCLC histology: a review of two Phase III studies. Oncologist 14, 253-263 (2009).

35. McLeod, H. L. et al. Pharmacokinetic and pharmacodynamic evaluation of the glycinamide ribonucleotide formyltransferase inhibitor AG2034. Clin. Cancer Res. 6, 2677-2684 (2000).

36. Yang, T. Y. et al. Sustained activation of ERK and Cdk2/cyclin-A signaling pathway by pemetrexed leading to S-phase arrest and apoptosis in human non-small cell lung cancer A549 cells. Eur. J. Pharmacol. 663, 17-26 (2011).

37. Yang, X., Matern, H. T. \& Gallwitz, D. Specific binding to a novel and essential Golgi membrane protein (Yip1p) functionally links the transport GTPases Ypt1p and Ypt31p. EMBO J. 17, 4954-4963 (1998).

38. Lee, E. et al. GRP78 as a novel predictor of responsiveness to chemotherapy in breast cancer. Cancer Res. 66, 7849-7853 (2006).

39. Cotter, T. G. Apoptosis and cancer: the genesis of a research field. Nat. Rev. Cancer 9, 501-507 (2009).

40. Simova, S., Klima, M., Cermak, L., Sourkova, V. \& Andera, L. Arf and Rho GAP adapter protein ARAP1 participates in the mobilization of TRAIL-R1/DR4 to the plasma membrane. Apoptosis 13, 423-436 (2008).

41. Kojima, Y. et al. Importin beta1 protein-mediated nuclear localization of death receptor 5 (DR5) limits DR5/tumor necrosis factor (TNF)-related apoptosisinducing ligand (TRAIL)-induced cell death of human tumor cells. J. Biol. Chem. 286, 43383-43393 (2011).

42. Zhang, X. D., Franco, A. V., Nguyen, T., Gray, C. P. \& Hersey, P. Differential localization and regulation of death and decoy receptors for TNF-related apoptosis-inducing ligand (TRAIL) in human melanoma cells. J. Immunol. 164, 3961-3970 (2000).

43. Akazawa, Y. et al. Death receptor 5 internalization is required for lysosomal permeabilization by TRAIL in malignant liver cell lines. Gastroenterology 136, 2365-2376 e2361-2367 (2009).

44. Hattula, K., Furuhjelm, J., Arffman, A. \& Peranen, J. A Rab8-specific GDP/GTP exchange factor is involved in actin remodeling and polarized membrane transport. Mol. Biol. Cell 13, 3268-3280 (2002).

45. Yoshimura, S., Gerondopoulos, A., Linford, A., Rigden, D. J. \& Barr, F. A. Familywide characterization of the DENN domain Rab GDP-GTP exchange factors. J. Cell Biol. 191, 367-381 (2010).

46. Luo, H. R. et al. GRAB: a physiologic guanine nucleotide exchange factor for Rab3A, which interacts with inositol hexakisphosphate kinase. Neuron 31, 439-451 (2001).

47. Bravo-Cordero, J. J. et al. MT1-MMP proinvasive activity is regulated by a novel Rab8-dependent exocytic pathway. EMBO J. 26, 1499-1510 (2007).

48. Moritz, O. L. et al. Mutant rab8 Impairs docking and fusion of rhodopsinbearing post-Golgi membranes and causes cell death of transgenic Xenopus rods. Mol. Biol. Cell 12, 2341-2351 (2001).

49. Sato, T. et al. The Rab8 GTPase regulates apical protein localization in intestinal cells. Nature 448, 366-369 (2007).

50. Faust, F., Gomez-Lazaro, M., Borta, H., Agricola, B. \& Schrader, M. Rab8 is involved in zymogen granule formation in pancreatic acinar AR42J cells. Traffic 9. 964-979 (2008) 
51. Caplan, S. et al. A tubular EHD1-containing compartment involved in the recycling of major histocompatibility complex class I molecules to the plasma membrane. EMBO J. 21, 2557-2567 (2002).

52. Roland, J. T., Kenworthy, A. K., Peranen, J., Caplan, S. \& Goldenring, J. R. Myosin $\mathrm{Vb}$ interacts with Rab8a on a tubular network containing EHD1 and EHD3. Mol. Biol. Cell 18, 2828-2837 (2007).
53. Jacobs, D. T., Weigert, R., Grode, K. D., Donaldson, J. G. \& Cheney, R. E. Myosin $\mathrm{Vc}$ is a molecular motor that functions in secretory granule trafficking. Mol. Biol. Cell 20, 4471-4488 (2009).

54. del Toro, D. et al. Mutant huntingtin impairs post-Golgi trafficking to lysosomes by delocalizing optineurin/Rab8 complex from the Golgi apparatus. Mol. Biol. Cell 20, 1478-1492 (2009). 